\title{
Féeries
}

Études sur le conte merveilleux, XVII ${ }^{\mathrm{e}} \mathrm{XIX} \mathrm{X}^{\mathrm{e}}$ siècle

$6 \mid 2009$

Le conte, les savoirs

\section{Tapis volants des Mille et Une Nuits}

The Thousand and One Night's Flying Carpets

Jean-Paul Sermain

\section{OpenEdition}

\section{Journals}

Édition électronique

URL : http://journals.openedition.org/feeries/713

DOI : $10.4000 /$ feeries. 713

ISSN : 1957-7753

Éditeur

UGA Éditions/Université Grenoble Alpes

Édition imprimée

Date de publication : 1 juillet 2009

Pagination : 179-188

ISBN : 978-2-84310-140-3

ISSN : $1766-2842$

Référence électronique

Jean-Paul Sermain, «Tapis volants des Mille et Une Nuits», Féeries [En ligne], 6 | 2009, mis en ligne le 15 septembre 2010, consulté le 07 septembre 2020. URL : http://journals.openedition.org/feeries/713 ; DOI : https://doi.org/10.4000/feeries.713

\section{(c) Féeries}




\section{Jean-Paul Sermain}

Université de la Sorbonne Nouvelle-Paris 3

\section{TAPIS VOLANTS DES MILLE ET UNE NUITS}

\section{Le tapis volant du prince Ahmed}

Au nom d'une version épurée de l'orientalisme, les nouveaux traducteurs des Mille et Une Nuits, J. E. Bencheikh et A. Miquel ont écarté, dans leur premier volume de la collection Folio (I99I), les histoires introduites dans la tradition par A. Galland ; elles sont d'ailleurs nommées « histoires orphelines " parce que sans aucune parentèle dans l'ensemble des recueils ou manuscrits arabes. Ce refus a ensuite été considérablement adouci dans l'édition complète de la collection Pléiade un peu ultérieure (2005), puisque la préface y est curieusement dédiée à Galland, dont est reconnu le rôle majeur dans le sauvetage de l'œuvre, marginalisée alors dans son lieu d'origine, et sont données en appendice non toutes les histoires orphelines mais les plus connues, l'Histoire d'Aladdin et l'Histoire d'Ali Baba. L'ostracisme affiché invoquait ces histoires et également deux symboles occidentaux de l'imaginaire oriental, la lampe et les tapis volants :

Il nous a semblé nécessaire de réagir contre les habitudes occidentales solidement établies. On ne trouvera ici ni Aladin, ni Ali Baba, ni Sindbâd, qui sont devenus les représentants trop connus d'un Orient fictif, peuplé de tapis volants et de lampes merveilleuses. On nous pardonnera de ne pas sacrifier au rituel ${ }^{\mathrm{I}}$.

Les histoires dont Galland est le responsable auraient donné de l'Orient une représentation fictive, redoublant ainsi le statut du genre par une transformation fantaisiste de son univers, dont la lampe merveilleuse et le tapis volants seraient les emblèmes. Comme ces objets font entrer les héros dans un monde surnaturel et les affranchissent des contraintes de la réalité, en donnant la fortune ou en abolissant l'espace, le lecteur investirait son

I. E. J. Bencheikh et A. Miquel, Les Mille et Une Nuits, Paris, Gallimard, collection " Folio ", I99I, I, p. I9. Sans doute les deux éditeurs traducteurs n'ont pas les mêmes positions et on peut imaginer que l'hostilité affichée a porté assez de fruits pour qu'il soit loisible de revenir à une position de compromis, sans doute aussi exigée par la maison Gallimard. 
imagination sur ces objets qui le plongent dans des mondes eux aussi déliés de toute réalité arabe ${ }^{2}$. Cette identification de l'Orient avec la fiction faisait partie des lieux communs historiographiques des XVII ${ }^{\mathrm{e}}$ et XVIII ${ }^{\mathrm{e}}$ siècles ${ }^{3}$, Galland la partage, mais en valorisant cette tendance et la fiction elle-même. À l'intérieur du livre, elle serait soutenue, ou anticipée, par le rôle donné à des objets familiers, typiques d'un mode de vie oriental s'agissant du tapis, et utilisant le potentiel symbolique de ses propriétés matérielles (d'où leur persistance jusqu'à l'âge du cinéma de masse) : la lampe éclaire ce qui est caché et fermé, et le tapis nous invite à reposer et à glisser d'un monde à l'autre en se laissant porter par le vent. L'expérience du voyageur se confondrait avec celle du lecteur, mais ce serait finalement pour ne rien connaître du Moyen-Orient, lui substituer ce que nous souhaitons y voir dans une sorte de transport quasi érotique.

Dans les histoires venues de Galland, et finalement écartées des deux collections Folio et Pléiade, figure l'unique occurrence du tapis volant dans le recueil, au cours de l'Histoire du prince Ahmed. Non qu'il n'y ait pas d'autres voyages aériens dans le recueil arabe. Deux épisodes analogues l'utilisent dans l'Histoire de Noureddin et dans celle de Caramalzaman : des génies sont frappés par la beauté extraordinaire de deux jeunes gens et prélèvent le jeune homme pour un voyage aérien, ménageant ainsi une rencontre amoureuse, charnelle et féconde dans un cas, chaste et passionnée dans l'autre, qui est à l'origine d'une obsession et d'aventures complexes. Mais on ne sait rien sur les modalités de ce transport : magie pure. L'occident était par avance prêt à associer l'Orient à de tels épisodes aériens puisque Le Décaméron de Boccace l'avait fait dans la seule nouvelle ouverte sur le monde arabe du recueil. Héros de la nouvelle huit de la dixième journée, Saladin, qui a été reçu généreusement à Pavie par Messer Torello, lui rend son hospitalité quand il est fait prisonnier pendant les croisades à Alexandrie. Il apprend que sa femme restée à Pavie doit croire qu'il est mort et qu'elle peut se remarier, il l'installe dans un lit et sollicite un magicien pour le faire transporter instantanément à Pavie, juste avant la consommation fatale d'un second mariage. Le changement apporté par le récit de Galland à une telle vision est de lier au voyage aérien le tapis

2. L'occident substituerait à un monde qu'il ignore, méprise ou manipule, ce qu'il veut bien imaginer, et profiterait même de l'éloignement pour en faire un champ de création fantasmatique d'une liberté sexuelle et cruelle sans limites. On en est bien loin chez Galland, auquel est fait le reproche inverse de censurer la sensualité arabe.

3. La meilleure expression, et nuancée, de cette identification est due à Huet dans son essai Sur l'origine des romans de 1670. 
oriental. Le prince Ahmed avec ses deux frères doivent apporter à leur père " la rareté la plus extraordinaire et la plus singulière " (III, p. 268), et celui qui l'emportera obtiendra en mariage la princesse. Le prince Houssain, venu en Inde, est étonné par le prix exorbitant demandé par un marchand pour un tapis :

Il faut donc qu'il soit précieux par quelque endroit qui ne m'est pas connu. - Vous l'avez deviné, Seigneur, repartit le crieur, et vous en conviendrez quand vous saurez qu'en s'asseyant sur ce tapis, aussitôt on est transporté avec le tapis où l'on souhaite d'aller, et l'on s'y trouve presque dans le moment sans que l'on soit arrêté par aucun obstacle. (III, p. 27I)

Ce tapis aidera, avec la longue vue et la pomme médicament, à sauver la princesse, mais il disparaît vite de l'œuvre parce que le prince Ahmed, écarté, trouve finalement un meilleur parti et de nouvelles aventures avec la fée Péri Banou.

Le réel obstacle est effacé. Que vaut dans cette perspective un savoir par ailleurs revendiqué dans des notes ? Surtout chez des auteurs peu au fait des textes et du monde arabe ? Quand Galland lit le début des Mille et Un Jours traduits par Pétis de la Croix - peut-être réécrit par Lesage! -, il ne peut mettre en doute la compétence linguistique de Pétis, mais la connaissance des realia qui en sont le substrat:

Je lus chez le président d'Olède environ la moitié du premier volume des Mille et Un Jours, composé à l'imitation des Mille et Une Nuits, sous le nom de M. Pétis. Le premier conte que je lus, et qui faisait environ cette moitié de volume, était tiré de contes turcs, c'est-à-dire des Quarante vizirs, ouvrage écrit en turc. J'y trouvai un nombre d'endroits susceptibles de critiques de la part de ceux qui ont un peu de connaissance du Levant ${ }^{4}$.

Mais qui en a ? Et à quelles sources? Celles de Gueullette ou de Caylus seront d'abord La Bibliothèque orientale d'Heberlot, elle-même encore sur le modèle humaniste, donc pour ces conteurs l'information n'est pas même de seconde main, de troisième main.

4. Notre édition de référence est celle en trois volumes de la collection "GF Flammarion " (2004). Le texte de Galland se trouve dans le tome I, p. 442. Si Les Mille et Un Jours ont eu une audience régulière dans la France et l'Angleterre du XVIII ${ }^{e}$ siècle, ils frappent aujourd'hui par leur sécheresse, leur incapacité à donner une vérité sensible à des situations dont le caractère stéréotypé s'en trouve un peu accusé. 


\section{Le tapis volant du critique}

Le critique attentif au savoir en matière des contes entend lire à contresens la fiction orientale : celle-ci réaliserait de façon hyperbolique son essence qui consiste à créer des mondes et à vous absorber en elle, usant de tapis pour vous éloigner de toute réalité, vous la faire survoler et oublier ses contraintes dans des rêveries débridées. La littérature d'imagination doit répondre à l'époque - aujourd'hui encore ? - de son écart avec les textes sérieux qui offrent des connaissances historiques ou morales et il faut beaucoup d'ingéniosité pour dresser de frêles ponts sur cette faille sans fond. Les notes fournissent alors d'utiles boucliers's. Mais le critique moderne, ainsi entièrement atteint par le soupçon anti-fictionnel de la première modernité (de Boccace à Diderot si l'on veut), se retrouve lui aussi sur un tapis volant bien déconcertant, et doué de propriétés encore plus variées que celui du prince Ahmed. En effet, ce tapis est naturellement extensible. Quelles limites donner au "savoir " inclus dans un texte de fiction étranger à la culture du lecteur ? Le texte de Galland signale par des notes ou par des commentaires des éléments étrangers au point de devenir incompréhensibles et exigeant une glose : ce qui est évident, invisible, banal, en Orient est au contraire surprenant pour des Français. Ainsi dès le deuxième paragraphe de la première Histoire (qui devient le cadre d'un premier cycle), celle du marchand et du génie, Galland détaille les gestes du héros après son repas : "il se lava les mains, le visage et les pieds, et fit sa prière » (p. 45), mais il ajoute " comme il était bon musulman » - détail qui explique la raison de ces gestes et informe le lecteur sur les rites religieux « là-bas ». De façon plus explicite encore, quand Ali-Baba, à la mort de son frère propose immédiatement de prendre sa veuve comme seconde épouse, le conteur ici sort de son propre monde pour s'adresser directement au lecteur français et prévenir son indignation ou un soupçon d'invraisemblance ; une fois le mariage fait, il ajoute : "Et comme ces

5. L'éditeur des Mille et Un Jours s'en avise et insère cet avis avant le $37^{\mathrm{e}}$ jour, faisant l'éloge de l'auteur : " Les savants auraient tort de lui reprocher d'avoir employé ses heures perdues à de pures bagatelles, puisque ces contes sont aussi utiles qu'agréables. En effet, on y marque exactement la géographie, on y peint les mœurs et les coutumes des différents peuples de l'Asie. Si la scène d'un conte est chez les Tartares, par exemple, on sait qu'on y vit autrement qu'à Bagdad ou en Égypte. [...] de plus, le traducteur y a joint une infinité de remarques curieuses; il a pris toutes les précautions imaginables pour qu'on lui pardonne son amusement et sauver, s'il se peut, cet ouvrage du mépris de certains lecteurs sérieux, qui ne sauraient souffrir les fictions les plus ingénieuses et à qui le plus agréable livre ne saurait plaire si le crédit de la vérité ne le rend recommandable. " (F. Pétis de la Croix, Les Mille et Un Jours, éd. P. Sebag, Paris, Phébus, 2003) 
sortes de mariages ne sont pas extraordinaires dans notre religion, personne n'en fut surpris. » (III, p. I94)

Sans cesse le conte fait appel à une expérience humaine générale : quand les voleurs découpent le cadavre de Cassim en quatre quartiers et les disposent de part et d'autre de la porte, c'est " pour épouvanter quiconque aurait la hardiesse de faire une pareille entreprise » (p. I88) : cruauté arabe ? cruauté humaine ? cruauté de truands? Ils ne comptent revenir qu'une fois révolu le délai de la putréfaction, "après que la puanteur sera exhalée ", mais Ali Baba arrive très vite sans qu'il soit rien dit de son malaise éventuel. Quand il apprend à sa belle sœur la mort de Cassim, son frère, et qu'il lui propose sans délai de la prendre pour seconde épouse, elle essuie vite ses larmes, "en supprimant les cris perçants ordinaires aux femmes qui ont perdu leur mari " : l'indécision de l'énonciation, à la fois celle du conteur arabe et celle du traducteur français, interdit de savoir s'il y a référence aux modes du deuil oriental ou à un trait féminin universel.

Il n'y a pas que le merveilleux qui déborde le savoir de la représentation. Dans la même histoire d'Ali Baba, le corps cousu de Cassim en fait partie, confié à un cordonnier dans l'obscurité, et pourtant tel qu'il peut en conclure : "J'ai cousu un mort. " Ici le souci du détail concret, l'intervention d'un artisan dûment caractérisé (par son âge), les yeux bandés, soupçonnant et voyant tout, l'enchaînement des actions banales de l'enterrement, dissimulent ou renforcent, c'est selon le point de vue critique, l'invraisemblance de l'épisode : mais que vaut alors un récit aussi soigneux de ses enchainements et de ses circonstances? Le critique est-il déjà là sur un tapis volant?

Du moins peut-il s'en tailler d'autres à sa guise, et de fort nombreux. Et de fort riches. Les travaux sur le texte arabe des Mille et Une Nuits reconstituent les différentes encyclopédies dans lesquelles il puise ou qu'il fait transparaître ou reconnaître : religieuses, historiques, économiques, géographiques, politiques, sociales, etc. Les Voyages de Sindbad sont à cet égard particulièrement fertiles et laisseraient voir en filigrane " des traces d'ouvrages arabes consacrés à la géographie ${ }^{6} "$. Des noms propres, des mesures, des événements permettent de dater sinon une histoire du moins sa reprise. De tels repérages peuvent être au service de recherches historiques ou culturelles. Il est par contre difficile de décider dans quelle mesure elles sont voulues par un auteur aussi évanescent que multiple dans des

6. Les Mille et Une Nuits, collection "Pléiade ", vol. II p. 999. Le bagage savant des deux traducteurs est d'ailleurs resté à la porte de cette édition, très pauvre en informations et sur le travail d'établissement du texte et sur les contextes orientaux. Nécessité économique ? 
contextes de lecture dont le seul à peu près reconstituable est celui des éditions du XIX ${ }^{e}$ siècle. L'étendue du tapis où repose le critique et son survol des textes sont donc toujours variables et pour une ouvre sans auteur et sans histoire pour le moins incertains, car si le savoir sous-jacent, affleurant dans le texte et parfois utilisé, est avéré, comment dire à quelles intentions, à quels effets pour le lecteur ? Comptait-on sur un savoir implicite? Mais cela concerne-t-il une littérature de divertissement dont le propos pourrait être ailleurs et relèverait plutôt de la philosophie morale. Ce qui intéresserait éventuellement le public arabe ne touche que de très loin le public français, venant plusieurs siècles après, avec d'autres références et ignorant presque tout du monde arabo-musulman. Mais là, un traducteur a été responsable de l'entreprise et l'a signée. Que voulait-il ?

\section{Le tapis volant d'Antoine Galland}

On a vu que Galland n'hésite pas à confier à son conteur arabe le souci de son public français : il parle de "notre " habitude orientale à un lecteur qui l'ignore et à qui il faut expliquer en quoi consistent des mœurs étrangères. Dans son Avertissement bien connu, Galland énumère les mérites des Mille et Une Nuits et, après le plaisir que procure l'art du récit, avant un profit moral hypothétique, il annonce des informations sur un monde inconnu : Les Mille et Une Nuits tiennent lieu d'un voyage alors fort fatigant, et même sont capables de fournir des connaissances très supérieures. Ces contes

doivent plaire encore par les coutumes et les mœurs des Orientaux, par les cérémonies de leur religion, tant païenne que mahométane ; et ces choses y sont mieux marquées que dans les auteurs qui en ont écrit et dans les relations des voyageurs. Tous ces Orientaux, Persans, Tartares et Indiens, s'y font distinguer, et paraissent tels qu'ils sont, depuis les souverains jusqu'aux personnes de la plus basse condition. Ainsi, sans avoir essuyé la fatigue d'aller chercher ces peuples dans leur pays, le lecteur aura ici le plaisir de les voir agir et de les entendre parler. On a pris soin de conserver leurs caractères, de ne pas s'éloigner de leurs expressions et de leurs sentiments » (I, p. 2I-22).

Galland voit dans les Nuits un moyen idéal de mettre en œuvre le projet de ses ouvrages antérieurs : faire connaître qui sont les Orientaux, comment ils vivent et ce qu'ils pensent. Qu'on puisse utiliser pour cela la fiction avait été défendu par Chapelain en faveur des vieux romans, et par les Modernes, Marivaux le formule de façon un peu provocante pour les textes antiques comme ceux d'Homère : s'il est vain d'en tirer des leçons littéraires (parce que ces leçons seraient mauvaises et, de toute façon, l'écri- 
vain véritable n'en a pas besoin), ils nous font découvrir d'autres manières de penser et même d'autres formes de sensibilité ${ }^{\text {. }}$.

L'Avertissement de Galland repère les enseignements à tirer des représentations (comme pour les cérémonies religieuses), mais « les mœurs et les coutumes " se manifestent plutôt dans les actions, dans les comportements des personnages, dans la matière même des contes : ce que le premier héros du livre emporte en voyage d'affaires, comment dans le désert un arbre invite à une pause déjeuner, et celle-ci composée de dattes, avec les noyaux jetés au loin. Il n'est pour cela nul besoin de notes ou de commentaires : voyez les vivre!

Galland va plus loin : le voyageur ne peut enregistrer que ce qu'il voit, et dont les motifs ou le sens lui échappent souvent ; ici les personnages et leurs actions ont été créés par les auteurs arabes, conformément à leurs expériences ; les " expressions » et les " sentiments » sont ceux des personnages : c'est leur vie intérieure, leur façon de penser et de sentir, leur imagination qui se manifestent dans ces discours, et ils sont tels que les auteurs les ont eux-mêmes conçus. L'important n'est pas qu'ils soient exactement conformes à ce que les Arabes feraient dans la réalité (leur vraisemblance impeccable) mais à ce qu'ont imaginé les auteurs, à la manière dont ils ont voulu représenter leur monde et qui a pu paraître intéressante et juste au public arabe : le texte montre la manière dont les auteurs inventent leurs héros et les font parler et s'émouvoir. Au-delà des coutumes, des façons habituelles de vivre, et qui sont d'autant plus intéressantes qu'elles vont de soi, qu'elles s'oublient presque dans des automatismes intégrés, la fiction nous donne à entrer dans les replis de la psychologie des peuples, dans ce qu'ils souhaitent à un moment donné projeter d'eux-mêmes dans des représentations attractives et touchantes. Aucun voyageur ne se mettra à la place des Arabes. La fiction permet cette expression vraie parce qu'elle n'est pas organisée et pensée pour un public étranger, elle n'est pas consciente d'elle-même et de ce projet. Le traducteur s'immisce dans l'interaction des représentations et des expressions arabes : il y place au centre le lecteur français, il le fait spectateur d'une scène qui n'a pas été peinte pour lui et qui tire de là sa vérité, sa signification. Cette connaissance passe par un partage des émotions et des intérêts : le lecteur français va admirer les contes, s'en étonner, entrer dans les fictions, se laisser ravir par leur langage. Faut-il en extraire une encyclopédie amorcée dans les

7. Marivaux, Journaux et æuvres diverses, éd. F. Deloffre et M. Gilot, Paris, Classiques Garnier, 1988, « Le Miroir » (1755), p. 534-549. 
notes? Galland est très sensible à l'origine ancienne de ces contes contemporains des anciens romans et les ayant nourris : rien dans sa traduction ne donne le moindre signal de cette profondeur temporelle. Rien ne permet au lecteur de repérer clairement les différents peuples ou pays orientaux, rien pour s'orienter dans la géographie des Nuits; on n'apprendra rien sur le calife historique Haroun al Raschid mis sur le même plan que des rois de fantaisie. Galland n'incite pas son lecteur à devenir savant de cette façon ${ }^{8}$.

\section{Le tapis volant du lecteur}

Et pourtant, comme l'a bien étudié C. Bahier-Porte', Galland insère des notes (cette pratique se développe au XVIII ${ }^{\mathrm{e}}$ siècle, devient monstrueuse, atteignant même les poèmes comme Les Saisons de Saint-Lambert) : à quel profit pour le lecteur? Quel tapis volant lui est-il ainsi taillé par Galland ? Ou bien le tapis volant de la lecture, comme le soupçonne Bencheikh, ne devait-il pas l'éloigner de la bibliothèque orientale ? Les notes demandent un moment qu'on s'abstraie d'histoires pourtant caractérisées par leur emprise exceptionnelle; Galland les utilise peu, elles sont rares et brèves, ponctuelles. Elles fournissent parfois une traduction littérale des noms propres. Comme l'ont fait remarquer Miquel et Bencheikh, un tel procédé est particulièrement égarant. Ils dénoncent ainsi Mardrus qui fait figurer de telles traductions dans le corps du texte, s'en sert pour désigner les personnages :

Un certain orientalisme alors à la mode le pousse [...] à traduire en français des noms de héros ou héroïnes, des noms qui en arabe, ont perdu toute valeur sémantique : un peu comme si, en français, nous disions " Homme " au lieu d' " André ", « Forestière " pour « Sylvaine », ou, pour " Christophe », « Porte-Christ » ${ }^{\text {10 }} .$.

Les notes fournissent ensuite des précisions géographiques, elles sont particulièrement nombreuses dans les Voyages de Sindbad, c'est-à-dire dans les voyages qui sont étrangers à tout souci de vraisemblance (alors que beaucoup de voyages dans les Nuits sont anodins). Quelques notes de

8. Cervantès dans son prologue à Don Quichotte se moque d'un savoir injecté dans le roman, d'autorités ou de citations, d'allusions. La fiction est utile autrement.

9. Christelle Bahier-Porte, "Les notes dans les premiers contes orientaux ", Féeries, n 2, 20042005, p. 9I-I08.

Io. Collection "Pléiade ", éd. cit., t. I, p. Xur. Exemple dans Les Mille et Une Nuits, t. II, p. 196 : " la princesse Haïatalnefous : ce mot est arabe et signifie la vie des âmes ". 
littérature (au sens de l'époque, de connaissances érudites des lettres) : par exemple le rapprochement du cyclope rencontré par Sindbad avec celui d'Homère. Et deux fois à peu de distance, dans l'Histoire de Caramalzaman, un renvoi à un roman médiéval : "Il y a dans le roman de Pierre de Provence et de la belle Maguelonne, une aventure semblable qui a été prise de celle-ci. » (II, p. I90 ; autre référence, p. 203)

Enfin, dernier exemple, d'allégation de coutume pour justifier un comportement. Dans l'Histoire de Noureddin Ali, le jeune Bedreddin, transporté dans les airs, se retrouve dans le lit de celle qu'il ignore être sa cousine et invité à " bien faire son devoir " d'époux. Pour cela, "il se déshabilla promptement. Il mit son habit sur un siège et sur la bourse que le juif lui avait donnée, laquelle était encore pleine, malgré tout ce qu'il en avait tiré. Il ôta son turban pour en prendre un de nuit qu'on avait préparé, il alla se coucher en chemise et en caleçon ». La note apparaît un peu superflue qui ajoute : "Tous les Orientaux couchent en caleçon et cette circonstance est nécessaire pour la suite" (I, p. 324). Les Occidentaux dorment alors nus. La traduction littérale du manuscrit Galland par Haddawy ignore ce caleçon :

She had no trousers on, so Badr-al-Din took off his trousers [...]. Then taking off his turban, which he laid over the wrapping cloth on the seat, he remained only in his shirt and skullcap and stood hesitating ${ }^{\mathrm{II}}$.

La nuit suivante, quand les génies l'emportent, il est de nouveau précisé qu'il ne porte que sa chemise sans pantalon; et quand il est déposé dans le cimetière et que le vent soulève sa chemise, tout son corps apparaît : "The breeze blew and raised his shirt, revealing legs and thighs and belly and navel as clear as chrystal and softer than cream ${ }^{12}$. "Galland opère un petit déplacement qui accentue même la beauté de la scène : "Un petit vent qui commençait alors à souffler leva sa chemise, et laissa voir sa poitrine qui était plus blanche que la neige. Ils furent tous tellement étonnés de cette blancheur...» (I, p. 325)

Essayons d'éclairer le rôle des notes à partir de ce dernier exemple. La note offre une information superflue puisqu'il importe peu de savoir si Bedreddin à ce moment là suit la coutume arabe ou non : l'essentiel est qu'il soit prêt pour la nuit d'amour et qu'ensuite son corps se révèle. Le

II. Husain Haddawy, The Arabian Nights, London, Everyman's Library, 1992, p. 178. La traduction Bencheikh Miquel est similaire.

I2. Ibid., p. I8o. La traduction Miquel Bencheikh et celle de Mardrus mentionnent explicitement le dévoilement du sexe. 
savoir sur les mœurs orientales (développé dans son livre Smyrne ancienne et moderne) embarrasserait ici plutôt la lecture s'il ne s'agissait pas pour Galland de conforter l'opération d'habillage du héros et du passage, pour éviter de rendre trop présente l'union charnelle et l'indécence du vent à Damas : la note sert ici à déguiser une manipulation ou plutôt participe de sa stratégie. Elle vise à mettre le texte " en bon français ».

Il en va ainsi pour les autres types de notes : celle concernant les noms réintroduisent des dénominations figurées qui seraient le propre de ce qu'on appelle alors le "style oriental " et que Galland a choisi d'écarter complètement de sa traduction, le considérant un obstacle pour goûter la beauté des contes ${ }^{13}$. Il en va de même pour les deux autres types de notes. La référence au roman médiéval est de peu d'intérêt pour des lecteurs qui sont coupés de leur passé médiéval; surtout elle sert à mentionner l'origine ancienne des contes, reconnue par Galland dans sa correspondance, alors même que Galland s'est gardé de laisser la moindre trace de cet ancrage temporel et il considère même que la peinture des mœurs restait valable, inentamée par le temps. Quant aux informations géographiques dans un contexte fantaisiste, elles impliquent aussi que les contes n'ont rient à nous apprendre sur ce plan là : qui ira les vérifier, qui s'en souviendra, qui ouvre pour cela Les Mille et Une Nuits?

Les notes, assez économes rappelons-le, servent donc à Galland non à donner des informations mais à dire qu'elles ne sont pas là, qu'il n'y en a pas, ou plus exactement qu'elles sont étrangères au recueil et à son propos : elles entrent dans une stratégie d'évitement, d'effacement, de la crudité du texte, de ses figures asiatiques, de son passé médiéval et de sa géographie fantaisiste ; elles permettent de faire apparaître le principe poétique de l'ouvrage et surtout de sa traduction, et, en figurant ponctuellement et inutilement ce que Galland aurait pu faire, elles signalent qu'il ne le fait pas : il n'écrira pas un texte orné, archaïque, sensuel, informatif; il fera l'exact contraire de Mardrus et de Burton. Les notes légères et désinvoltes maintiennent au contraire le lecteur sur le tapis volant de la fiction orientale, en lui faisant fugacement entrevoir tout ce qu'il laisse de côté dans ce voyage aérien.

I3. On n'en trouve trace que dans le conte particulier d'Aboulhassan : histoire d'amour pathétique qui donne une grande place aux échanges galants oraux et écrits des deux amants. 